\title{
Myocardial remodeling, an overview
}

\author{
Dennis V. Cokkinos $\cdot$ Costas Pantos
}

Published online: 26 September 2010

(C) Springer Science+Business Media, LLC 2010

Myocardial remodeling (REM) is a deleterious process characterized by gradual cardiac enlargement, cardiac dysfunction and typical molecular changes. It is a universal phenomenon, being caused by many pathological conditions $[1,2]$.

Of these, myocardial infarction is the more common. It has been estimated that even with the timely use of primary angioplasty, and with the subsequent use of all the currently recommended drug therapies, which will be discussed later, the emergence of REM is around 30\%, eventually leading to heart failure and death $[3,4]$. It has been stressed that if $20 \%$ of the "myocardium at risk" is salvaged, the course toward heart failure can be avoided [5].

Moreover, uncontrolled hypertension is still a major cause of heart failure, evolving to REM. It must not be forgotten that valvular heart disease, even with the progress of cardiac surgery, can still lead to cardiac dilatation, effectively REM.

Only very recently it was stated that in mitral regurgitation, operation before the left ventricular end-systolic diameter exceeds $40 \mathrm{~mm}$, survival is higher [6]. REM is also an end result of cardiomyopathy, either post-myocarditis or genetically produced, and cancer chemotherapy [7].

D. V. Cokkinos $(\bowtie)$

Biomedical Research Foundation, Academy of Athens, Onassis Cardiac Surgery Center, 356 Sygrou Ave, Kallithea,

17674 Athens, Greece

e-mail: dcokkinos@bioacademy.gr

\section{Pantos}

Department of Pharmacology, University of Athens,

75 Mikras Asias Ave, Goudi, 11527 Athens, Greece
The term was first introduced by Janice Pfeffer's group in 1985 [8].

A comprehensive review was given 4 years ago by Opie et al. [9]. One part of the 1990 definition which they re-iterate is very important, i.e. it represents an important therapeutic target. REM must be viewed as a process that is initially "adaptive"; aiming at offsetting an unfavorable situation. Thus, in aortic stenosis and hypertension, the ensuing left ventricular concentric hypertrophy results in reduced myocardial wall stress; conversely, in the course, in mitral and aortic regurgitation, left ventricular eccentric hypertrophy concerns stroke volume conservation. Thus, hypertrophy finally leads to ventricular dilatation and becomes "maladaptive" and detrimental [10].

However, it is not only the myocardium that undergoes pathological changes. It is currently widely recognized that two more elements contribute to REM and cardiac malfunction:

- The development of myocardial fibrosis, resulting from increased abnormal collagen production. Fibrosis results in systolic but also diastolic dysfunction through increased cardiac stiffness [11].

- The inadequate increase in capillary density of the hypertrophying and remodeling myocardium that results in inadequate oxygenation [12]. Indeed, even after an acute myocardial infarction, capillary density is decreased as REM ensues [13]

As already mentioned, a main characteristic of the remodeling myocardium is the return to the "fetal" phenotype, which is characterized by decreased contractility but also lower energy consumption [14]. Also, the ratio HMC $\alpha / \beta$ is decreased, ANP and BNP and $\alpha$-actin overexpressed, and the SERCA/phospholamban activity decreased. 
The eminent research group of Taegtmeyer et al. stresses the increase point that the fetal myocardium is more resistant against necrosis [15]. A parallel paradigm is myocardial hibernation, in which diminished performance is offset by persistence of viability [16].

However, the fetal myocardium as an evolutionary model is dysfunctional.

Baker et al. have shown that immature hearts cannot be preconditioned [17].

Moreover, Krenz and Robbins, in a model to transgenic mice overexpressing $\beta$-MHC, have shown a more unfavorable post-infarct course [18]. Proteomic [19] and genomic [20] analyses have been recently carried out. Very recently, MiR423-5p has been described as involved in heart failure [21], while MiR $133 \alpha$ is protective against cardiac fibrosis [22]. The number of serum markers that also reflect myocardial concentrations is rapidly increasing. Galectin-3 is acquiring prominence as a marker of fibrosis and heart failure progression [23].

ST-2 has recently been considered as a specific marker of REM [24]. Myostatin is also a marker of hypertrophy [25] and heart failure [26].

As already stated, continuous efforts are aimed at the prevention and regression of REM. These efforts are either invasive or pharmacological [27, 28].

However, the main thrust is the combination of the two approaches of which Table 1 gives only an approximation.

It is not surprising that many journals are devoting whole issues to REM [1].

In this issue of "Heart Failure Reviews", we attempt a necessarily incomplete account of molecular aspects of REM, with frequent references to therapeutic approaches. Nabeebaccus, Zhang and Shah from King's College in London provide an account of the NADPH oxidases in REM. These oxidases are significant sources of ROS and particularly important in redox signaling. The two main isoforms in the expressed in the heart are NOX2 and NOX4. The authors point that some of the main families in the treatment of heart failure such as ACE inhibitors are ARBs and statins, which act through NOX2 inhibition. Additionally, they reply that the modulation of NOX activity may lead to new therapeutic targets.

Gajarsa and Kloner from the Harbor-UCLA Medical Center in Torrance and the University of Southern California, Los Angeles, California examine the neurohormonal activity of the renin-angiotensin-aldosterone axis and its molecular effects on the myocardium.

They point out the importance of the extracellular matrix and the matrix metalloproteinases (MMPs) and their tissue inhibitor counterparts. The many transcription factors regulating MMP activity are discussed. Trials involving selective and broad MMP inhibitors or TIMP activators are discussed.
Table 1 Approaches to prevention and regression of remodeling

(A) Pharmacological

(1) During ischemia reperfusion

Adenosine, nicorandil, ANP, statins, cyclosporin

Post-conditioning opioids

(2) Long term

(a) Proposed through animal data

Valproic acid (HDAC inhibitor)

(b) Metabolic modulators

Exenatide, insulino

Mimetic

Tropic

Etomoxir, trimetazidine, ranolazine

MAPK activators- AICAR (exercise mimetic?)

PPAR activators

Metformin

Clenbuterol ( $\beta 2$ receptor agonist)

Tetracyclines ( $\uparrow$ SERCA)

(c) Hormones-peptides

Thyroid hormones

Growth hormone

Estradiol

Testosterone

(d) Antioxidants-quercetin, resveratrol, curcumin

Erythropoietin

Atrial and brain natriuretic peptide Neseritide

(e) Miscellaneous

Sildenafil

Rapamycin (mTor inhibitor

Interleukin receptor antagonists (anakinra)

(f) Clinically used

$\beta$-blockers, ACE inhibitors-angiotensin receptor blockers, aliskiren, statins, aldosterone inhibitors

(B) Interventional-surgical-electrophysiological

Exercise

Progenitor cell infusion

Cardiac shock wave therapy

Biventricular pacing (cardiac resynchronization)

Early reperfusion (thrombolysis, primary angioplasty)

Left ventricular reconstruction surgery

Left ventricular assist devices

Cell-based therapy and mechanical assist devices and their molecular effects are described.

The aspects of myocardial necrosis and replacement fibrosis are discussed by Gandhi et al. from Weber's team from the University of Tennessee in Memphis. The authors stress that replacement fibrosis appears at areas of previous necrosis. It initially plays the role of preserving the structural integrity of the myocardium, but it subsequently has adverse 
functional consequences contributing to progressive heart failure. Intracellular $\mathrm{Ca}^{2+}$ overloading and induction of oxidative-predominantly mitochondrialstress are involved in cardiomyocyte necrosis.

Pro-oxidant $\mathrm{Ca}^{2+}$ overloading is counterbalanced by a intrinsically coupled $\mathrm{Zn}^{2+}$ entry, which acts as an antioxidant. A simple cardioprotective strategy might involve adjuvant nutriceuticals. Hyposelenemia and its association with dilated cardiomyopathy is also discussed.

The role of STAT3 that is involved in cardiomyocyte protection and hypertrophy are discussed by Haghikia, Stapel, Hoch and Hilfiker-Kleiner from the Medical School of Hannover. This protein also impacts on the vasculature and extracellular matrix through paracrine pathways.

Additionally, it is also a mitochondrial protein involved in energy protection. STAT3 is activated by many factors, i.e. cytokine growth factors, neurohormones, mechanical load and ischemia. They point out possible therapeutic targets and the need of satisfactory control, since persistent activation of STAT3 in other organs may produce malignant de-differentiation and tumor growth, but can also be involved in vascular remodeling and arterial neointimal hyperplasiaanother aspect of the yin and yang concept [29].

The role of the innate immune system is amply covered in two articles.

Petra Kleinbongard, Schulz and Heusch from the University Clinic of Essen discuss the role of $\mathrm{TNF} \alpha$ in myocardial ischemia/reperfusion, remodeling and heart failure. This cachexin is part of the response to different forms of stress. Myocardial TNF $\alpha$ and its receptor activation have an ambivalent role:

Excessive TNF $\alpha$ expression and receptor type 1 activation induce contractile dysfunction, hypertrophy, fibrosis and cell death, while lower concentration and stimulation are protective.

The conflicting results of $\mathrm{TNF} \alpha$ antagonist trials with etanercept and infliximab are discussed. Potential explanations are given. They stress that a selective suppression of TNFR 1 activation or a selective activation of TNFR2 may give better results.

Another aspect of innate immunity and its effect on REM is provided by Guro Valen from the University of Oslo. She points out that innate immunity is expressed in the injured heart as cytokine release from the heart, activation of toll-like receptors and the transcription factor nuclear factor Kappa B. However, although activation immunity is actually detrimental in acute inflammation, the long-term outcomes in in vivo models of hypertrophy and REM are less clear. Activation of innate immunity may provide a beneficial process counteracting the vicious cycle of heart failure development. Thus, TLR signaling may reduce hypertrophic response and cardiac fibrosis, while
TLR2-deficient mice had less REM after an experimental myocardial infarction.

Finally Pantos, Mourouzis and Cokkinos from the University and Biomedical Research Foundation of the Academy of Athens describe the role of thyroid hormones (TH) in REM. They point out that an experimental myocardial infarction or pressure overload decrease $\mathrm{TH}$ signaling and activate the fetal gene program in the heart. TH administration limits reperfusion injury and prevents or induces regression of REM by favorably affecting cardiac chamber geometry in a time-dependent manner and may convert pathological to physiological hypertrophy. They re-iterate the fact that although earlier and small clinical studies have provided favorable results, the efficiency of $\mathrm{TH}$ in large clinical trials in the treatment of REM and heart failure still await evaluation.

The above cited articles do not pretend to cover the whole range of REM. A larger publication in the form of a book might be logically perspective. However, all the contributions in this issue of "Heart Failure Reviews" are provided by groups long immersed in their respective fields of expertise. They provide an up to date and hopefully detailed view of this fascinating and clinically very important aspect.

\section{References}

1. Diez J, Ertl GA (2009) Translational approach to myocardial remodelling. Cardiovasc Res 81:409-411

2. Hill JA, Olson EN (2008) Cardiac plasticity. N Engl J Med 358:1370-1380

3. Savoye C, Equine O, Tricot O et al (2006) Left ventricular remodelling after anterior wall acute myocardial infarction in modern clinical practice (from the Remodelage Ventriculaire [REVE] study group. Am J Cardiol 98:1144-1149

4. Papadopoulos CE, Karvounis HI, Giannakoulas G et al (2007) Predictors of left ventricular remodelling after reperfused acute myocardial infarction. Am J Cardiol 99:1024-1025

5. Miura T, Miki T (2008) Limitation of myocardial infarct size in the clinical setting: current status and challenges in translating animal experiments into clinical therapy. Basic Res Cardiol 103:501-513

6. Tribouilloy C, Grigioni G, Avierinos J-F et al (2009) Survival implications of left ventricular end-systolic diameter in mitral regurgitation due to flail leaflets: a long-term follow-up multicenter study. J Am Coll Cardiol 54:1961-1968

7. Yeh ETH, Bickford Cl (2009) Cardiovascular complications of cancer therapy. J Am Coll 53:2231-2247

8. Pfeffer JM, Pfeffer MA, Braunwald E (1985) Influence on chronic captopril theraphy on the infarcted left ventricle of the rat. Circ Res 57:84-95

9. Opie LH, Commerford RJ, Gersh BJ et al (2006) Controversies in ventricular remodeling. Lancet 367:356-367

10. Dorn GW II, Robbins J, Sugden PH (2003) Phenotyping hypertrophy: eschew obfuscation. Circ Res 92:171-1175

11. Burak M, Frangogiannis NG (2007) The role of TGF- $\beta$ signaling in myocardial infarction and cardiac remodeling. Cardiovasc Res 74:184-195 
12. Frey N, Olson EN (2003) Cardiac hypertrophy: the good, the bad and the ugly. Annu Rev Physiol 65:45-79

13. Westenbrink BD, Ruifrok W-PT, Voors AA et al (2010) Vascular endothelial growth factor is crucial for erythropoietin-induced improvement of cardiac function in heart failure. Cardiovasc Res 87:30-39

14. Swynghedaw B (2006) Phenotypic plasticity of adult myocardium. Molecular mechanisms. J Exp Biol 209:2320-2327

15. Rajabi M, Kassiotis C, Razeghi P, Taegtmeyer H (2007) Return to the fetal gene program protects the stressed heart: a strong hypothesis. Heart Fail Rev 12:331-343

16. Heusch G, Schulz R, Rahimtoola SH (2005) Myocardial hibernation-a delicate balance. Am J Physiol Heart Circ Physiol 288:H984-H999

17. Baker JE, Holman P, Gross GJ (1999) Preconditioning in immature rabbit hearts: role of KATP channels. Circulation 99: $1249-1254$

18. Krenz M, Robbins J (2004) Impact of beta-myosin heavy chain expression on cardiac function during stress. J Am Coll Cardiol 44:2390-2397

19. Cleniewski-Bernard C, Mulder P, Henry JP et al (2008) Proteomic analysis of left ventricular remodeling in an experimental model of heart failure. J Proteome Res 7:5004-5016

20. Sarwar R, Copok SA (2009) Genomic analysis of left ventricular remodelling. Circulation 120:437-444

21. Tijsen AJ, Creemers EE, Moerland PD et al (2010) MiR423-5p as a circulating biomarker for heart failure: questions about MiR423-5p. Circ Res 106:1035-1039
22. Matkovich SJ, Wang W, Tu Y et al (2010) MicroRNA-133a protects against myocardial fibrosis and modulates electrical repolarization without affecting hypertrophy in pressure-overload adult hearts. Circ Res 106:166-175

23. De Boer RA, Voors AA, Muntendam P et al (2009) Galectin-3: a novel mediator of heart failure development progression. Eur $\mathrm{J}$ Heart Fail 11:811-817

24. Weir RAP, Miller AM, Murphy GEJ et al (2010) Soluble ST2 is marker for predicting post-MI recovery. J Am Coll Cardiol 55:243-250

25. Shyu KG, Lu MJ, Wang BM et al (2006) Myostatin expression in ventricular myocardium in rat model of volume-overload heart failure. Eur J Clin Invest 36:713-719

26. George I, Bish LT, Kamalakkannan G et al (2010) Myostatin activation in patients with advanced heart failure and after mechanical unloading. Eur J Heart Fail 12:420-422

27. Ishii H, Amano T, Matsubara T, Myrohara T et al (2008) Pharmacological intervention for prevention of left ventricular remodeling and improving prognosis in myocardial infarction. Circulation 118:2710-2718

28. Bolognese L, Nescovic AN, Parodi G et al (2002) Left ventricular remodeling after primary coronary angioplasty: patterns of left ventricular dilation and long-term prognostic implications. Circulation 106:2351-2357

29. Cokkinos DV (2004) Preconditioning - a paradigm of yin and yang. Hellenic J Cardiol 43:179-182 